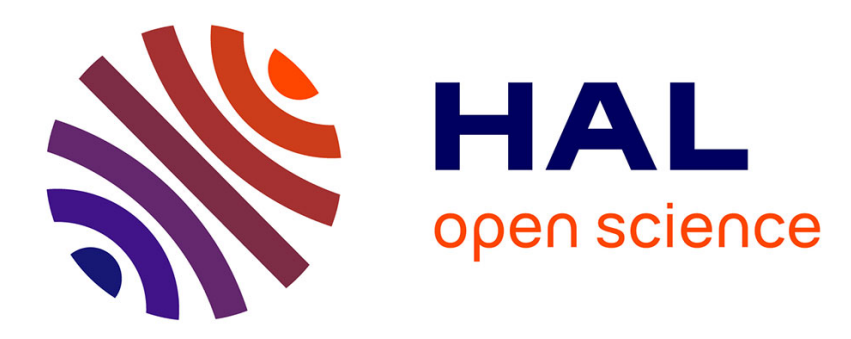

\title{
Mesure des forces d'oscillateur dans le spectre du Néon I, par étude de la dispersion anormale \\ M. Crance
}

\section{To cite this version:}

M. Crance. Mesure des forces d'oscillateur dans le spectre du Néon I, par étude de la dispersion anormale. Revue de Physique Appliquée, 1973, 8 (4), pp.325-331. 10.1051/rphysap:0197300804032500 . jpa-00243690

\section{HAL Id: jpa-00243690 https://hal.science/jpa-00243690}

Submitted on 1 Jan 1973

HAL is a multi-disciplinary open access archive for the deposit and dissemination of scientific research documents, whether they are published or not. The documents may come from teaching and research institutions in France or abroad, or from public or private research centers.
L'archive ouverte pluridisciplinaire HAL, est destinée au dépôt et à la diffusion de documents scientifiques de niveau recherche, publiés ou non, émanant des établissements d'enseignement et de recherche français ou étrangers, des laboratoires publics ou privés. 
Classification

Physics Abstracts :

$5.230-5.235$

\title{
MESURE DES FORCES D'OSCILLATEUR DANS LE SPECTRE DU NÉON I, PAR ÉTUDE DE LA DISPERSION ANORMALE
}

\author{
M. CRANCE \\ Laboratoire Aimé-Cotton, CNRS II, Bâtiment 505, 91405 Orsay, France
}

(Reçu le 10 juillet 1973)

\begin{abstract}
Résumé. - Nous avons réalisé une expérience de mesure de forces d'oscillateur relatives, par une méthode dérivée de la méthode des crochets de Rozhdestvenskii, qui est fondée sur l'étude de la dispersion anormale au voisinage d'une raie d'absorption. Nous effectuons, par un enregistrement photoélectrique, l'analyse spectrale de la figure d'interférence d'un interféromètre de Michelson éclairé en lumière blanche dont un bras contient la vapeur à étudier. Afin d'éprouver la méthode, nous avons mesuré les forces d'oscillateur de quelques transitions visibles du néon I $\left(2 p^{5} 3 s-2 p^{5} 3 p\right)$ pour lesquelles on dispose de nombreuses mesures antérieures et de valeurs théoriques précises.

Abstract. - A method derived from the Rozhdestvenskii hook method is described. It is based on the study of anomalous dispersion close to an absorption line and it is used to measure relative oscillator strengths. Here one arm of a Michelson interferometer holds the vapor to be studied. Using a white light source the interferometer produces an interference pattern which is analyzed by a photoelectric spectrometrer. In order to test the experiment, we have measured the oscillator strengths of some visible neon I transitions $\left(2 p^{5} 3 s-2 p^{5} 3 p\right)$ for which many former measurements and precise theoretical values are available.
\end{abstract}

1. Introduction. - Les probabilités de transition radiative dans les spectres atomiques peuvent être atteintes soit en émission, soit en absorption. La comparaison de l'intensité de diverses raies spectrales émises à des longueurs d'onde très différentes, rencontre les difficultés inhérentes à toute mesure photométrique absolue. Quant aux études d'absorption elles nécessitent une bonne connaissance des profils de la raie d'absorption et de la source utilisée ce qui pose souvent de délicats problèmes. Cependant, cette difficulté peut être levée par l'étude de la dispersion anormale directement liée à l'absorption. Une méthode particulièrement bien adaptée à l'étude de la dispersion anormale a été proposée par Rozhdestvenskii en 1912. En effet dans la méthode des crochets, la quantité mesurée n'est ni une intensité ni un indice, mais une longueur d'onde. Cependant dans sa forme classique le champ d'application de cette méthode est limité par l'emploi des plaques photographiques qui ne sont pas de bons récepteurs dans l'infrarouge. Pour éviter cette limitation nous avons transposé la méthode de manière à pouvoir utiliser un récepteur photoélectrique. C'est cette adaptation que nous décrivons dans le présent article.

Dans le premier paragraphe nous rappelons les points essentiels de la méthode de mesure des forces d'oscillateur par l'étude de la dispersion anormale. Puis nous décrivons le montage expérimental en accordant une attention particulière à la description et à l'exploitation du signal obtenu. Les avantages de la méthode photographique sont ensuite discutés. Enfin, nous présentons les résultats que nous avons obtenus pour quelques transitions $2 p^{5} 3 s-2 p^{5} 3 p d u$ néon I. La précision de nos mesures est évaluée à $5 \%$ ce qui est confirmé par la comparaison de nos résultats avec des valeurs obtenues par une tout autre méthode et avec le résultat de calculs théoriques particulièrement raffinés [3]-[6].

2. Principe de la méthode. - L'étude de la dispersion anormale d'une vapeur au voisinage d'une raie d'absorption permet, en principe, la détermination de la force d'oscillateur de la transition. Loin d'une raie d'absorption l'indice d'une vapeur est en effet donné par la formule de Kramers-Heisenberg [2]

$$
n-1=\frac{e^{2}}{2 \pi m c^{2}} \sum_{j>i}\left(N_{i}-N_{j} \frac{g_{i}}{g_{j}}\right) \frac{f_{i j}}{\sigma_{i j}^{2}-\sigma^{2}} .
$$

La double sommation est faite sur tous les niveaux de l'atome, classés par ordre d'énergies croissantes.

$N_{i}$ est la population du niveau $i, g_{i}$ sa multiplicité, $\sigma_{i j}$ est le nombre d'onde de la transition entre les niveaux $i$ et $j ; f_{i j}$ la force d'oscillateur de cette transition.

Cette formule n'est pas valable pour les nombres d'onde tels que $\left|\sigma-\sigma_{0}\right|$ soit de l'ordre de la largeur $\gamma$ de la raie d'absorption mais nous avons toujours travaillé dans des conditions telles que $\left|\sigma-\sigma_{0}\right|$ soit 
supérieur à $\gamma$. Par ailleurs si l'on considère une raie d'absorption bien isolée et si tout en respectant la condition ci-dessus, on travaille au voisinage de cette raie, alors les termes dus aux autres transitions varient assez lentement pour qu'on puisse les considérer comme constants. En conséquence, si on se limite au domaine de nombres d'onde tels que

$$
\gamma<\left|\sigma-\sigma_{0}\right| \ll \sigma
$$

on peut représenter la variation de l'indice par la fonction $n(\sigma)$ :

$$
n(\sigma)-n_{0}=\frac{e^{2}}{4 \pi m c^{2} \sigma} \frac{f_{i j}}{\sigma_{0}-\sigma}\left(N_{i}-N_{j} \frac{g_{i}}{g_{j}}\right) .
$$

On tiendrait compte de l'effet Doppler en faisant le produit de convolution de $n(\sigma)$ et d'une gaussienne de largeur $\Delta \sigma_{\mathrm{D}}\left(\Delta \sigma_{\mathrm{D}}\right.$ étant la largeur Doppler de la raie d'absorption) ; toutefois dans "la région où sont faites les mesures, la variation de l'indice est assez lente sur une largeur Doppler pour qu'on puisse conserver cette forme de l'indice (dans le cas que nous avons étudié, l'erreur relative faite sur l'indice est de l'ordre de $10^{-3}$ à $1 \mathrm{~cm}^{-1}$ du centre de la raie d'absorption).

Pour étudier l'indice et ses variations nous utilisons le fait que la présence d'un gaz excité dans l'un des bras d'un Michelson éclairé en lumière blanche modifie son pouvoir de transmission. L'interféromètre est réglé de manière à donner des anneaux à l'infini dont on étudie la partie centrale. En l'absence de vapeur, on établit une différence de chemin optique $x$ entre les deux bras de l'interféromètre. La vapeur est introduite dans le bras le plus court. Si on définit par $l / 2$ l'épaisseur de vapeur traversée, la différence de chemin optique entre les deux ondes interférant au centre des anneaux est $\Delta=(n-1) l-x$. Le pouvoir de transmission $T(\sigma)$ pour la radiation de nombres d'onde $\sigma$, au centre des anneaux est égale à

$$
T_{0}(1+\cos 2 \pi \sigma \Delta)=T_{\mathrm{c}}(1+\cos \varphi) .
$$

En utilisant la formule simplifiée de l'indice et en posant

$A=\frac{e^{2} f_{i j} l}{4 \pi m c^{2}}\left(N_{i}-N_{j} \frac{g_{i}}{g_{j}}\right) \quad$ et $\quad x^{\prime}=x-\left(n_{0}-1\right) l$

on obtient

$$
\varphi=2 \pi \sigma \Delta=2 \pi\left(\frac{A}{\sigma_{0}-\sigma}-\sigma x^{\prime}\right) .
$$

La fonction $T(\sigma)$ présente des extremums d'une part quand $\varphi=k \pi$ ( $k$ entier), d'autre part quand $\varphi$ est stationnaire, c'est-à-dire lorsque $\mathrm{d} \varphi / \mathrm{d} \sigma=0$.

$$
\frac{\mathrm{d} \varphi}{\mathrm{d} \sigma}=2 \pi\left(x^{\prime}-\frac{A}{\left(\sigma_{0}-\sigma\right)^{2}}\right)
$$

est nul pour $\sigma$ tel que $\left(\sigma-\sigma_{0}\right)^{2}=A / x^{\prime}$.

Pour $\left|\sigma-\sigma_{0}\right| \gg \sqrt{A / x^{\prime}}, T(\sigma)$ est une sinusoïde, pour les deux valeurs $\sigma_{0} \pm \sqrt{A / x^{\prime}}, T(\sigma)$ est station- naire. Puis quand $\left|\sigma-\sigma_{0}\right|$ diminue, la phase varie très vite avec $\sigma, T(\sigma)$ se met à osciller rapidement. On étudie le pouvoir de transmission du Michelson à l'aide d'un spectromètre à haute résolution.

La mesure des nombres d'onde pour lesquels $T(\sigma)$ est stationnaire et la mesure de la période de la sinusoïde donnent accès au produit

$$
\left(N_{i}-N_{j} \frac{g_{i}}{g_{j}}\right) f_{i j}
$$

et dans le cas où $N_{i} \ll N_{j}$, à $N_{j} f_{i j}$. L'étude d'un couple de transitions, de même niveau inférieur permet de mesurer le rapport de leurs forces d'oscillateur.

3. Dispositif expérimental. - Le dispositif expérimental est schématisé dans la figure 1 . On remarque qu'il est constitué essentiellement par un interféromètre de Michelson, un spectromètre à réseau et un dispositif d'asservissement et de détection. Nous décrivons successivement ces trois parties.

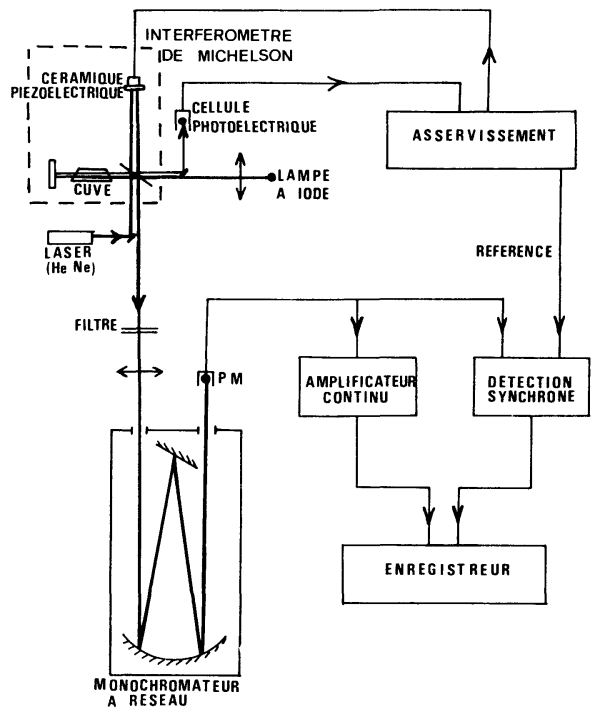

Fig. 1. - Dispositif expérimental.

3.1 INTERFÉROMÈTRE. - Nous utilisons un interféromètre de Michelson de configuration classique. Les fenêtres du tube qui contient la vapeur à étudier, taillées dans le même verre que la séparatrice ont des épaisseurs choisies de manière à jouer le rôle d'une compensatrice. Celle-ci étant imparfaitement réglée, la différence de chemin optique entre les deux bras de l'interféromètre dépend de la longueur d'onde. D'autre part, indépendamment de la dispersion anormale, l'indice moyen $n_{0}$ de la vapeur dépend de la raie étudiée. $x^{\prime}$ dépend donc de la longueur d'onde mais présente une variation négligeable sur les quelques $\AA$ où nous étudions l'indice au voisinage d'une transition.

L'interféromètre est éclairé en lumière blanche. La source de lumière blanche est une lampe quartz-iode, placée au foyer d'une lentille. La lampe est alimentée par un courant régulé, qui lui assure une brillance extrêmement stable. 
La vapeur étudiée dans l'expérience décrite ici est du néon à une pression de quelques torr contenu dans un tube de $50 \mathrm{~cm}$ de long et $1 \mathrm{~cm}$ de diamètre. Le gaz est excité par une décharge continue ; le courant est stabilisé réglable de 0 à $30 \mathrm{~mA}$.

3. 2 Spectromètre. - La lumière issue du Michelson est analysée par un spectromètre formé d'un monochromateur et d'un photomultiplicateur placé derrière la fente de sortie. Le monochromateur, de focale $2 \mathrm{~m}$ est équipé d'un réseau à 270 traits par $\mathrm{mm}$, de $10 \mathrm{~cm} \times 20 \mathrm{~cm}$ dont l'angle de miroitement égal à $67^{\circ}$ correspond à $\lambda=6 \mu$ dans le premier ordre. Les transitions visibles sont observées dans un ordre élevé ; nous utilisons une série de filtres interférentiels pour isoler la bande étudiée. Le monochromateur est équipé de fentes. Dans la direction parallèle aux fentes, l'étendue géométrique utilisée est limitée par la cuve. L'angle maximum sur l'axe du tube d'un rayon utile est $5 \times 10^{-3}$ rad ce qui correspond à une variation d'ordre d'interférence d'environ $1 / 20$ pour une différence de chemin optique de $2 \mathrm{~mm}$ entre les bras de l'interféromètre. Ceci ne diminue pratiquement pas le contraste du spectre cannelé et ne déforme pas la figure enregistrée. Dans la direction perpendiculaire aux fentes, l'étendue géométrique du faisceau utile est limitée par le monochromateur ; quand on augmente la résolution, on diminue le signal utile et donc le rapport signal sur bruit. Ceci nous a conduit à prendre des fentes de même largeur $(\lesssim 100 \mu)$ ce qui correspond à une résolution d'environ 50000 . Pour que le spectre cannelé soit observable avec un bon contraste il faut alors que la différence de chemin optique entre les deux bras de l'interféromètre n'excède pas 4 à $5 \mathrm{~mm}$. La distance des extremums est d'autant plus grande que $x^{\prime}$ est plus petit, mais pour qu'on puisse considérer la raie étudiée comme isolée il faut que les extremums restent beaucoup plus près de cette raie que des raies voisines. Afin que les effets dus aux raies voisines soient négligeables, de même que les variations de la dispersion des lames, nous n'avons pas utilisé de différence de chemin optique entre les bras de l'interféromètre inférieure à $0,1 \mathrm{~mm}$. Afin d'obtenir un bon rapport signal sur bruit nous avons utilisé un photomultiplicateur à couche trialcaline, particulièrement bien adapté à l'étude des transitions

\section{du néon $\mathrm{I}$.}

$$
2 p^{5} 3 s-2 p^{5} 3 p
$$

3.3 ASSERVISSEMENT ET DÉTECTION. - L'état d'interférence et par conséquent la différence de marche de l'interféromètre doivent être extrêmement stables. De très faibles variations de la température de l'air changent beaucoup le chemin optique. Pour compenser les variations de l'indice de l'air, nous avons réalisé un système d'asservissement de la différence de chemin optique entre les deux bras de l'interféromètre. Le faisceau lumineux issu d'un laser $\mathrm{HeNe}$ à $6328 \AA$ traverse l'interféromètre, le faisceau sortant du
Michelson est recueilli sur une cellule photoélectrique. Le miroir appartenant au bras qui ne contient pas la vapeur est monté sur une céramique piézo-électrique à laquelle on applique une tension alternative à une fréquence de $800 \mathrm{~Hz}$ qui lui donne un déplacement total de l'ordre de $3 \mu$. Pour asservir l'appareil sur un minimum de transmission à $6328 \AA$ on superpose à la tension alternative une tension continue telle que la composante à $800 \mathrm{~Hz}$ du signal soit annulée.

3.4 DesCRIPTION DU SignAL. - La lumière que reçoit le photomultiplicateur est formée du spectre cannelé dû à la lumière blanche et du spectre émis par le tube à néon. Il est nécessaire d'étudier le spectre cannelé seul. Comme les raies émises par le tube à néon sont assez intenses, les ghosts d'une raie et les pieds de la fonction d'appareil, bien que très faibles en valeur relative, perturbent beaucoup le spectre cannelé. La différence de chemin optique dans l'interféromètre est modulée, on fait une détection synchrone de cette modulation, ce qui fournit un signal proportionnel à $\sin 2 \pi\left(x^{\prime} \sigma+\frac{A}{\sigma-\sigma_{0}}\right)$ (cf. Annexe). C'est sur ce signal que nous étudions la dispersion anormale de la vapeur. Le système de rotation du réseau permet d'obtenir sur la fente de sortie du monochromateur un défilement du spectre étudié, linéaire en nombre d'onde. Nous enregistrons simultanément le signal issu de la détection synchrone et le signal continu reçu par le photomultiplicateur et amplifié. La figure 2 montre un exemple d'enregistrement. Loin d'une raie d'absorption nous obtenons une sinusoïde, puis les deux extremums; dans la partie du spectre où la phase varie très vite (entre les extremums) la résolution devient insuffisante pour permettre d'obtenir un bon contraste, on observe, entre les extremums, l'amorce d'une sinusoïde, puis la valeur moyenne du sinus, c'est-à-dire zéro. Pour déterminer la position des extremums on utilise la propriété suivante : si pour deux nombres d'onde $\sigma^{\prime}$ et $\sigma^{\prime \prime}$ de part et d'autre d'un extremum la phase du sinus est la même, alors

$$
\left(\sigma^{\prime}-\sigma_{0}\right)\left(\sigma^{\prime \prime}-\sigma_{0}\right)=\frac{A}{x^{\prime}}
$$

La partie continue du signal permet de déterminer le centre de la raie d'absorption, qui coïncide avec le centre de la raie émise par le tube.

4. Comparaison des méthodes photographique et photoélectrique. - La méthode des crochets mise en œuvre par Rozhdestvenskii en 1912 [1] est fondée sur le même principe : interféromètre à deux ondes éclairé en lumière blanche, croisé avec un spectromètre. Un des bras de l'interféromètre contient la vapeur à étudier, l'autre bras contient une lame qui introduit une différence de chemin optique entre les deux bras. On forme des franges d'interférence perpendiculaires à la fente d'entrée d'un spectrographe et on photographie le spectre. (Pour un nombre d'onde l'ordre d'inter- 

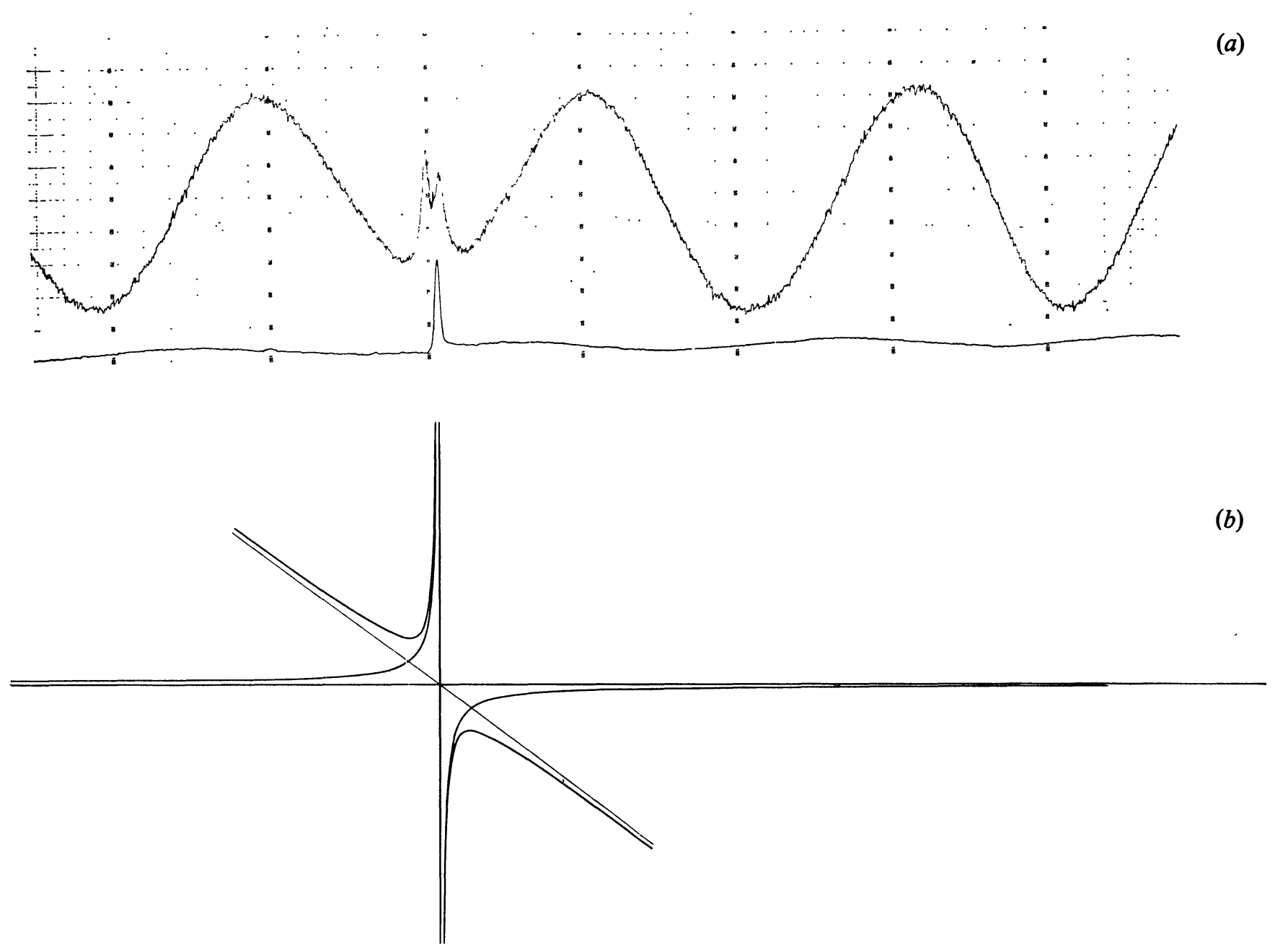

FIG. 2.

a. - Spectre cannelé au voisinage de $\lambda=6096 \AA$ (l'interfrange $1 / x$, vaut $17 \mathrm{~cm}^{-1}$ ).

$b$. - Variation en fonction du nombre d'onde, de l'indice de la vapeur et de la différence de chemin optique entre les deux bras de l'interféromètre dans les conditions de l'enregistrement.

férence varie linéairement le long de la fente). On obtient sur la plaque du spectrographe des courbes de même éclairement, correspondant à un même ordre d'interférence, elles sont de la forme

$$
y(\sigma)=x \sigma+\frac{A}{\sigma-\sigma_{0}} .
$$

Elles présentent des extremums traditionnellement appelés « crochets » à une distance proportionnelle à $\sqrt{\left(N_{i}-N_{j} \frac{g_{i}}{g_{j}}\right) f_{i j}}$ de la raie d'absorption.

Les deux méthodes donnent accès à la même information. L'avantage des plaques photographiques est essentiellement multiplex, ce qui se manifeste sous deux formes :

- on étudie simultanément plusieurs transitions,

- pour chaque nombre d'onde on observe une variation continue de l'état d'interférence le long de la fente d'entrée du spectromètre.
Cependant le premier point est d'un intérêt limité puisque la valeur optimale de la différence de marche dans l'interféromètre n'est pas la même pour toutes les transitions et il est nécessaire de prendre plusieurs photographies avec des différences de marche différentes. De plus le second point ne sert qu'à repérer les ordres d'interférence entiers. Sur un enregistrement photoélectrique on observe un seul ordre d'interférence pour chaque nombre d'onde mais on peut choisir $x^{\prime}$ de manière que les extremums se produisent pour des valeurs du sinus proches de zéro, ce qui correspond aux meilleures conditions d'observation des extremums. L'enregistrement contient alors toutes les informations qu'on peut obtenir d'une plaque photographique.

En outre la méthode photoélectrique apporte deux améliorations supplémentaires :

— possibilité d'étudier des transitions infrarouges : il n'est pas possible actuellement de photographier des spectres pour des longueurs d'onde supérieures à 
I $\mu$ environ alors qu'il existe des cellules photoélectriques sensibles jusqu'à l'infrarouge lointain. Nous espérons atteindre des transitions que la méthode photographique ne permet pas d'étudier,

- augmentation de la précision : la précision sur la mesure de $N f$ donnée par Pery Thorne et Chamberlain est $12 \%$; dans la méthode photoélectrique l'incertitude est réduite à $5 \%$ (l'incertitude essentiellement due aux instabilités de l'interféromètre ne peut être que réduite dans l'infrarouge).

Les franges obtenues sur une plaque photographique sont sinusoïdales. Le principe de la mesure est tel qu'elles ne peuvent pas être rendues infiniment fines ce qui limite fondamentalement la précision avec laquelle est obtenue la courbe $y(x)$. Dans un enregistrement photoélectrique nous nous affranchissons de cette contrainte.

5. Résultats. - Nous avons étudié les transitions entre les configurations $2 p^{5} 3 s$ et $2 p^{5} 3 p$ du néon $I$. Ces transitions se situent entre $5400 \AA$ et $8000 \AA$. Les mesures montrent que les populations des niveaux de $2 \mathrm{p}^{5} 3$ s observées sont de l'ordre de $10^{12}$ atomes par $\mathrm{cm}^{3}$

$$
\begin{array}{ll}
\mathrm{s}_{5} & 0,9 \times 10^{12} \text { atomes par } \mathrm{cm}^{3} \\
\mathrm{~s}_{4} & 0,4 \times 10^{12} \text { atomes par } \mathrm{cm}^{3} \\
\mathrm{~s}_{3} & 0,9 \times 10^{11} \text { atomes par } \mathrm{cm}^{3} \\
\mathrm{~s}_{2} & 0,8 \times 10^{11} \text { atomes par } \mathrm{cm}^{3} .
\end{array}
$$

Les populations des quatre niveaux de $2 \mathrm{p}^{5} 3 \mathrm{~s}$ sont du même ordre de grandeur. Deux de ces niveaux $s_{5}\left({ }^{3} P_{2}\right)$ et $\mathrm{s}_{3}\left({ }^{3} \mathrm{P}_{0}\right)$ sont métastables vis-à-vis des transitions dipolaires électriques alors que $s_{4}\left({ }^{3} P_{1}\right)$ et $s_{2}\left({ }^{1} P_{1}\right)$ sont liés au niveau fondamental par des transitions dipolaires électriques intenses (le couplage n'est pas purement Russell-Saunders) et doivent leur forte population à l'emprisonnement de radiation qui en résulte.

Au-delà de $20 \mathrm{~mA}$ les populations des niveaux de la configuration $2 \mathrm{p}^{5} 3 \mathrm{~s}$ varient peu (quelques \% entre 20 et $30 \mathrm{~mA}$ ). Nos mesures ont été faites avec un courant de $30 \mathrm{~mA}$. Nous avons vu que l'étude des extremums de la figure d'interférence de part et d'autre d'une transition $\sigma_{0}$ entre les niveaux $i$ (supérieur) et $j$ (inférieur) nous donne la valeur de $\left(N_{i}-N_{j} \frac{g_{i}}{g_{j}}\right) f_{i j}$. Nous avons vérifié que $N_{i} \ll N_{j}$ pour les transitions étudiées. Quand le courant de décharge dans le tube passe de 20 à $30 \mathrm{~mA}$ l'intensité des transitions $2 \mathrm{p}^{5} 3 \mathrm{~s}-$ $2 \mathrm{p}^{5} 3 \mathrm{p}$ augmente, donc les populations des niveaux de $2 \mathrm{p}^{5} 3 \mathrm{p}$ augmentent ; les valeurs de $A$ augmentent très peu et pour un couple de transitions de même niveau inférieur le rapport $A_{i j} / A_{k j}$ ne varie pas ; ce qui nous amène à conclure que la condition $N_{i} \ll N_{j}$ est bien remplie et que nous mesurons $N_{j} f_{i j}$ pour chaque transition étudiée. Nous obtenons les valeurs relatives de forces d'oscillateur pour des transitions de même

\begin{tabular}{|c|c|c|c|c|c|c|}
\hline Transition & $\lambda(\AA)$ & (a) & [6] & [4] & [3] & [5] \\
\hline - & - & & & & & \\
\hline $\mathrm{s}_{5} \mathrm{p}_{2}$ & 5882 & 0,076 & 0,0764 & 0,206 & 0,132 & 0,072 \\
\hline $\mathrm{s}_{5} \mathrm{p}_{4}$ & 5945 & 0,15 & 0,133 & 0,257 & 0,181 & 0,136 \\
\hline $\mathrm{s}_{5} \mathrm{p}_{5}$ & 5976 & 0,021 & 0,0224 & - & & 0,026 \\
\hline $\mathrm{s}_{5} \mathrm{p}_{6}$ & 6143 & 0,32 & 0,358 & 0,474 & 0,370 & 0,370 \\
\hline$s_{5} p_{7}$ & 6217 & 0,066 & 0,0484 & 0,142 & 0,102 & 0,048 \\
\hline $\mathrm{s}_{5} \mathrm{p}_{8}$ & 6334 & 0,24 & 0,223 & 0,291 & 0,250 & 0,249 \\
\hline $\mathrm{s}_{5} \mathrm{p}_{9}$ & 6402 & 1 & 1 & 1 & 1 & 1 \\
\hline $\mathrm{s}_{5} \mathrm{p}_{10}$ & 7032 & 0,29 & 0,273 & 0,312 & 0,290 & 0,259 \\
\hline $\mathrm{s}_{4} \mathrm{p}_{2}$ & 6030 & 0,14 & 0,093 & & - & 0,089 \\
\hline $\mathrm{s}_{4} \mathrm{p}_{3}$ & 6074 & 0,46 & 0,326 & 0,796 & 0,40 & 0,341 \\
\hline $\mathrm{s}_{4} \mathrm{p}_{4}$ & 6096 & 0,64 & 0,511 & 0,90 & 0,613 & 0,527 \\
\hline $\mathrm{s}_{4} \mathrm{p}_{6}$ & 6304 & 0,18 & 0,125 & - & - & 0,134 \\
\hline $\mathrm{s}_{4} \mathrm{p}_{7}$ & 6382 & 0,62 & 0,615 & 0,732 & 0,709 & 0,612 \\
\hline $\mathrm{s}_{4} \mathrm{p}_{8}$ & 6506 & 1 & 1 & & & 1 \\
\hline$s_{4} p_{10}$ & 7245 & 0,26 & 0,254 & 0,425 & 0,394 & 0,250 \\
\hline$s_{3} p_{2}$ & 6164 & 0,67 & 0,595 & 0,809 & 0,613 & 0,537 \\
\hline $\mathrm{s}_{3} \mathrm{p}_{5}$ & 6266 & 1 & 1 & & & 1 \\
\hline $\mathrm{s}_{3} \mathrm{p}_{7}$ & 6533 & 0,54 & 0,476 & 0,670 & 0,610 & 0,481 \\
\hline$s_{2} p_{1}$ & 5852 & 0,56 & 0,451 & - & - & 0,469 \\
\hline $\mathrm{s}_{2} \mathrm{p}_{2}$ & 6599 & 0,66 & 0,555 & 一 & - & 0,570 \\
\hline $\mathrm{s}_{2} \mathrm{p}_{4}$ & 6678 & 1 & 1 & - & - & 1 \\
\hline $\mathbf{s}_{2} \mathbf{p}_{5}$ & 6717 & 0,62 & 0,593 & - & - & 0,570 \\
\hline $\mathrm{s}_{2} \mathrm{p}_{6}$ & 6929 & 0,87 & 0,858 & - & - & 0,811 \\
\hline
\end{tabular}
niveau inférieur. La valeur de $N f$ obtenue à partir d'un enregistrement se déduit de la mesure de 5 à
Forces de raie relatives

(a) : ce travail.

10 couples de points pour chaque extremum. Nous faisons la moyenne des valeurs obtenues; aucune ne s'écarte de plus de $5 \%$ de la moyenne. La reproductibilité des mesures de forces de raie relatives est bien compatible avec cette incertitude. Dans le tableau nous reproduisons nos résultats ainsi que ceux obtenus par la méthode des crochets par Ladenburg (1933) [3] et Pery Thorne et Chamberlain (1963) [4]. Nous avons également reporté les valeurs mesurées en émission par Bridges et Wiese [5] ainsi que les valeurs calculées par E. Koenig [6]. Nos résultats sont en général plus proches de ceux de Ladenburg que de ceux de Pery Thorne et Chamberlain [4] et ils sont en général compatibles aussi bien avec ceux de E. Koenig [6], qu'avec ceux de Bridges et Wiese [5].

6. Conclusion. - Nous avons mesuré un certain nombre de valeurs de $N f$ correspondant à des transitions $2 p^{5} 3 s-2 p^{5} 3 p$ du spectre du néon I. Celles-ci sont de l'ordre de $10^{10}$ à $10^{12} \mathrm{~cm}^{-3}$. Certaines transitions $2 p^{5} 3 s-2 p^{5} 3 p$ ont des forces d'oscillateur 10 à 100 fois plus faibles et ainsi nous n'avons pas été en mesure de les étudier. Pour la plupart de celles-ci nous avons observé néanmoins une déformation de la figure d'interférence mais la mesure n'a pu être faite avec une bonne précision.

Nous avons ainsi montré que la méthode des crochets photoélectrique apporte une amélioration dans la mesure des forces d'oscillateur relatives dans le visible et permet d'envisager une extension à l'étude des transitions infrarouges pour lesquelles la plaque photographique ne peut être utilisée.

Il est difficile de comparer les méthodes étudiant la dispersion anormale aux méthodes de mesures d'intensité qui ont donné de très bons résultats sur ces transitions du néon. Ces dernières sont difficiles à mettre en œuvre car elles exigent des mesures photométriques 
absolues. Les mesures de dispersion anormale et d'intensité sont complémentaires puisque les forces d'oscillateur mesurées sont dans un cas relatives à un même niveau inférieur, dans l'autre cas, à un même niveau supérieur.
Remerciements. - Cetté étude a été menée sous la direction du Professeur J. Brochard. Je remercie S. Liberman et J. Pinard des nombreux conseils et encouragements qu'ils m'ont prodigués tout au long de ce travail.

\section{ANNEXE}

Forme de la courbe enregistrée. - Un interféromètre de Michelson, éclairé par une source blanche contient dans l'un de ses bras la vapeur à étudier. Il est réglé de manière à former des anneaux à l'infini.

La différence de chemin optique entre les deux ondes interférant au centre des anneaux à l'infini est de la forme $x^{\prime}+\frac{A}{\sigma\left(\sigma-\sigma_{0}\right)}$. Le pouvoir de transmission du Michelson, pour la radiation de nombre d'onde $\sigma$ est :

$$
T(\sigma)=T_{0}\left(1+\cos 2 \pi\left(\left(x_{0}^{\prime}+\delta e \cos 2 \pi v t\right) \sigma+\frac{A}{\sigma-\sigma_{0}}\right)\right)
$$

où $v$ est la fréquence $(800 \mathrm{~Hz})$ de vibration du miroir mobile de l'interféromètre. La lumière est analysée par le monochromateur. L'intensité lumineuse reçue par le photomultiplicateur $T_{1}(\sigma)$ est le produit de convolution de $T(\sigma)$ par la fonction d'appareil du monochromateur, mais la diffraction n'intervenant pratiquement pas on a :

$$
T_{1}(\sigma)=\frac{1}{a^{2}} \int_{\sigma-a / 2}^{\sigma+a / 2} \mathrm{~d} \sigma^{\prime} \int_{\sigma^{\prime}-a / 2}^{\sigma^{\prime}+a / 2} \mathrm{~d} \sigma^{\prime \prime} T\left(\sigma^{\prime \prime}\right)
$$

$a$ largeur spectrale déterminée par la fente du monochromateur.

Le signal issu du photomultiplicateur est envoyé à une détection synchrone à la fréquence $v$ qui fournit la fonction enregistrée $f(\sigma)$.

$f(\sigma)$ est proportionnelle à la composante de Fourier de $T_{1}(\sigma)$ à la fréquence $v$, et celle-ci est obtenue à partir de la composante de Fourier de $T(\sigma)$.

COMPOSANTE DE FOURIER DE $T(\sigma)$ A FRÉQUENCE $v$.

$T(\sigma)=, 1+\cos 2 \pi\left(x_{0}^{\prime} \sigma+\frac{A}{\sigma-\sigma_{0}}\right) \cos (2 \pi \sigma \delta e \cos 2 \pi v t)-\sin 2 \pi\left(x_{0}^{\prime} \sigma+\frac{A}{\sigma-\sigma_{0}}\right) \sin (2 \pi \sigma \delta e \cos 2 \pi v t)$.

Le développement de Fourier de $(\cos 2 \pi v t)^{m}$ ne contient un terme à la fréquence $v$ que si $m$ est impair. La composante de $(\cos 2 \pi v t)^{2 n+1}$ à la fréquence $v$ est

$$
\frac{1}{2^{n}} C_{2 n+1}^{n} \cos 2 \pi v t
$$

Seul le développement de $\sin (2 \pi \sigma \delta e \cos 2 \pi v t)$ fournira une composante à la fréquence $v$ :

$$
\sin (2 \pi \sigma \delta e \cos 2 \pi v t)=\sum_{0}^{\infty}(-1)^{n} \frac{(2 \pi \sigma \delta e \cos 2 \pi v t)^{2 n+1}}{(2 n+1) !}
$$

dont la composante de Fourier à la fréquence $v$ est

$$
2 \cos 2 \pi v t \sum_{0}^{\infty}(-1)^{n}\left(\frac{2 \pi \sigma \delta e}{2}\right)^{2 n+1} \times \frac{1}{n !(n+1) !}
$$

c'est-à-dire $2 \cos \omega t J_{1}(2 \pi \sigma \delta e)$.

La composante de Fourier de $T(\sigma)$ à la fréquence $v$ est

$$
-2 \cos 2 \pi v t J_{1}(2 \pi \sigma \delta e) \sin 2 \pi\left(x_{0}^{\prime} \sigma+\frac{A}{\sigma-\sigma_{0}}\right) \text {. }
$$

Sur les quelques $\mathrm{cm}^{-1}$ observés au voisinage d'une raie d'absorption $\sigma$ varie peu, donc $J_{1}$ varie peu ; on peut admettre que $f(\sigma)$ est proportionnelle à

$$
\frac{1}{a^{2}} \int_{\sigma-a / 2}^{\sigma+a / 2} \mathrm{~d} \sigma^{\prime} \int_{\sigma^{\prime}-a / 2}^{\sigma^{\prime}+a / 2} \mathrm{~d} \sigma^{\prime \prime} \sin 2 \pi\left(x_{0}^{\prime} \sigma^{\prime \prime}+\frac{A}{\sigma^{\prime \prime}-\sigma_{0}}\right)
$$


$x_{0}^{\prime} \sigma \gg 1$ une très petite variation de $x_{0}^{\prime}$ fait donc beaucoup changer la valeur de $\sin 2 \pi\left(x_{0}^{\prime} \sigma+\frac{A}{\sigma-\sigma_{0}}\right)$

l'extremum observé est d'autant plus accusé que le sinus est voisin de zéro ; si c'est le cas, à l'extremum $\sigma_{1}$

et au voisinage de l'extremum

$$
x_{0}^{\prime} \sigma+\frac{A}{\sigma-\sigma_{0}}=x_{0}^{\prime} \sigma_{1}+\frac{A}{\sigma_{1}-\sigma_{0}}=\frac{\varphi_{1}}{2 \pi}
$$

$$
\sin 2 \pi\left(x_{0}^{\prime} \sigma+\frac{A}{\sigma-\sigma_{0}}\right) \quad \text { est peu différent de } \quad 2 \pi\left(x_{0}^{\prime} \sigma+\frac{A}{\sigma-\sigma_{0}}\right)-\varphi_{1}
$$

$f(\sigma)$ est peu différent de

$$
g(\sigma)=\frac{1}{a^{2}} \int_{\sigma-a / 2}^{\sigma+a / 2} \mathrm{~d} \sigma^{\prime} \int_{\sigma^{\prime}-a / 2}^{\sigma^{\prime}+a / 2} \mathrm{~d} \sigma^{\prime \prime}\left[2 \pi\left(x_{0}^{\prime} \sigma^{\prime \prime}+\frac{A}{\sigma^{\prime \prime}-\sigma_{0}}\right)-\varphi_{1}\right]
$$

$$
\begin{gathered}
g(\sigma)=2 \pi\left\{x_{0}^{\prime} \sigma-\varphi_{1}+\frac{A}{a^{2}}\left[\left(\sigma-\sigma_{0}+a\right) \log \left(\sigma-\sigma_{0}+a\right)+\left(\sigma-\sigma_{0}-a\right) \log \left(\sigma-\sigma_{0}-a\right)-2\left(\sigma-\sigma_{0}\right) \log \left(\sigma-\sigma_{0}\right)\right]\right\} \\
g^{\prime}(\sigma)=2 \pi\left[x_{0}^{\prime}+\frac{A}{a^{2}}\left(\log \frac{\left(\sigma-\sigma_{0}\right)^{2}-a^{2}}{\left(\sigma-\sigma_{0}\right)^{2}}\right)\right] .
\end{gathered}
$$

Un développement au second ordre par rapport à $a / \sigma-\sigma_{0}$ donne

$$
\begin{aligned}
& g(\sigma) \simeq 2 \pi\left[x_{0}^{\prime} \sigma+\frac{A}{\sigma-\sigma_{0}}\left(1+\frac{a^{2}}{6\left(\sigma-\sigma_{0}\right)^{2}}\right)\right]-\varphi_{1} \\
& g^{\prime}(\sigma) \simeq 2 \pi\left[x_{0}^{\prime}-\frac{A}{\left(\sigma-\sigma_{0}\right)^{2}}\left(1+\frac{a^{2}}{2\left(\sigma-\sigma_{0}\right)^{2}}\right)\right]
\end{aligned}
$$

les extremums de $g(\sigma)$ se produisent pour

$$
\left(\sigma-\sigma_{0}\right)^{2}=\frac{A}{x_{0}^{\prime}}\left(1+\frac{a^{2}}{2 A / x_{0}^{\prime}}\right) .
$$

Ceci montre que si on analyse la figure d'interférence avec une résolution trop faible on trouvera des extremums trop éloignés et on sera conduit à surestimer $N f$. L'erreur systématique ainsi introduite $\left(\frac{a^{2}}{2 A / x_{0}^{\prime}}\right.$ en valeur relative $)$ est de $1 \%$ dans le pire des cas.

\section{Bibliographie}

[1] RozhdestvenskiI, D. S., Annln der Phys. 39 (1912) 307.

[2] Korff, S. and Breit, G., Rev. Mod. Phys. 4 (1932) 471.

[3] Ladenburg, R., Rev. Mod. Phys. 5 (1933) 243.
[4] Pery Thorne, A. and Chamberlain, J. E., Proc. Phys. Soc. 82 (1963) 133.

[5] Bridges, J. M. and Wiese, W. L., Phys. Rev. 2 (1970) 285.

[6] Koenig, E., Phys. Lett. 34A (1971) 284. 